\title{
Implementation of an Algorithm for the Transfer of Citrus Using an Intelligent Model for Trains
}

\author{
Fernando García-Isidro ${ }^{1}$, Julissa Martinez-Lugo ${ }^{1}$, Alberto Ochoa ${ }^{2}$, \\ José Alberto Hernández Aguilar ${ }^{3}$ \\ ${ }^{1}$ Instituto Tecnológico de Superior de Naranjos, \\ Mexico \\ ${ }^{2}$ Maestría en Cómputo Aplicado, UACJ, \\ Mexico \\ ${ }^{3}$ FCAeI, Universidad Autónoma del Estado de Morelos, \\ Mexico
}

\begin{abstract}
We consider the implementation of an algorithm for the transfer of citrus using an intelligent model for trains, this research is performed based on the problem of packing in containers (Bin Packing), so that objects or boxes should be accommodated in a finite number of cubes considering to minimize the use of containers. The problem of packing containers will be understood and made according to the space of the container, the calculation will be made by maximizing the number of items - or boxes of citrus- that can be stored safely, the dimensions of the container shall also be addressed, taking into account that all have the same capacity and size, an algorithm based on MATLAB was used for the arrangement of objects in containers with containers crossing name is used.
\end{abstract}

Keywords: Container, Packaging, Trains, Capacity, Dimension, Objects, Bin Packing, MATLAB.

\section{Introduction}

For its simplicity and combinatorial nature of the problem of packaging container makes it an NP- hard problem, that is to say, that there is no algorithm that can solve all instances of the problem in a given polynomial time with respect to the number of input objects.

The problem of packing containers has great relevance for transporting, loading trains, trucks and cargo vehicles with weight capacity, distribution of tasks in homogeneous processes, and organization objects using a computer through blocks.

In order to solve the problem for a large number of objects it has been established several very simple algorithms such as: 
Next fit (AS): In this algorithm objects are listed, the first is assigned to the container number 1, the rest are assigned to the current container if fit, otherwise is assigned the next container, the new container becomes the current container.

First-fit algorithm (PA): In this algorithm, the objects are listed as containers, therefore the first object is assigned to the container 1, the rest of the objects are assigned to another container where they fit, and if the objects do not fit in any container which are assigned a new one is created.

Best Fit algorithm (MA): This algorithm is a modification of the AS for which the current object to the container having the minimum space available to store the object is assigned. In case of a tie between two or more containers, the one with the lowest risk is chosen.

In order to improve these three types of algorithms as a first step you can rearrange the objects depending on the space they occupy in descending order.

A typical example of the problem and its solution according Lodi A, S Martello, Vigo D are shown in Figures 1 and 2 respectively.

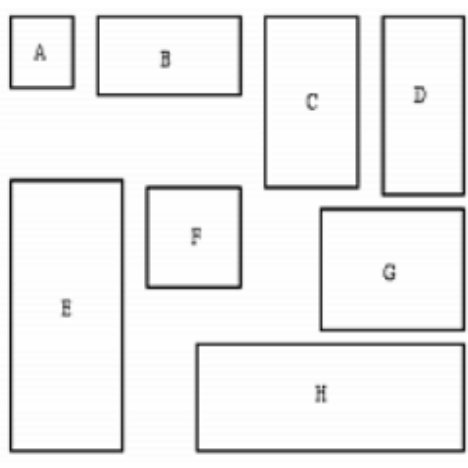

Fig. 1. Input.

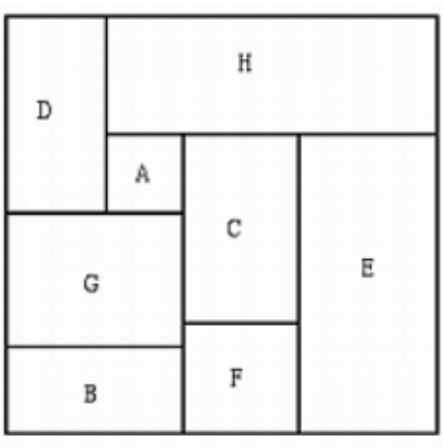

Fig. 2. Output.

Fig. 1. shows the objects (A, B, C, D, E, F, G, H) and observed that there are spaces between objects, on the other hand, Figure 2 shows a solution for this type of objects. [4]

For all types of wheeled vehicles able to run on a railway track are known as trains or rolling stock, which aims to transport different types of cargo.

Man's need to transport large amounts of cargo led him to build machines with great traction. George Stephenson built the first locomotive on 25 July of 1814, which later led to a railroad. At first locomotive it was used in coalmines where the drag of 40 tones was shown at a speed of 40 kilometers per hour.

The railroad was the product of the industrial revolution originated in England in the eighteenth and nineteenth centuries. The evolution of the locomotive has been remarkable, and its use has not only been for freight, but also for the transport of persons, both large and small distances.

The iron transport is the transport used worldwide. It offers a variety of different types of rail freight cars in boxcars, gondolas, hoppers, and trailer, duplex and triplex tank cars. 
The vans are used to transport products that require protection from the environment without temperature control.

Otherwise boxcars transport perishable goods with temperature control; gondolier transport materials that do not require protection from the environment; the hoppers are used for the transportation of industrial products such as coal, scrap metal, etc.

Loads and dimensions are stipulated in international standards [6].

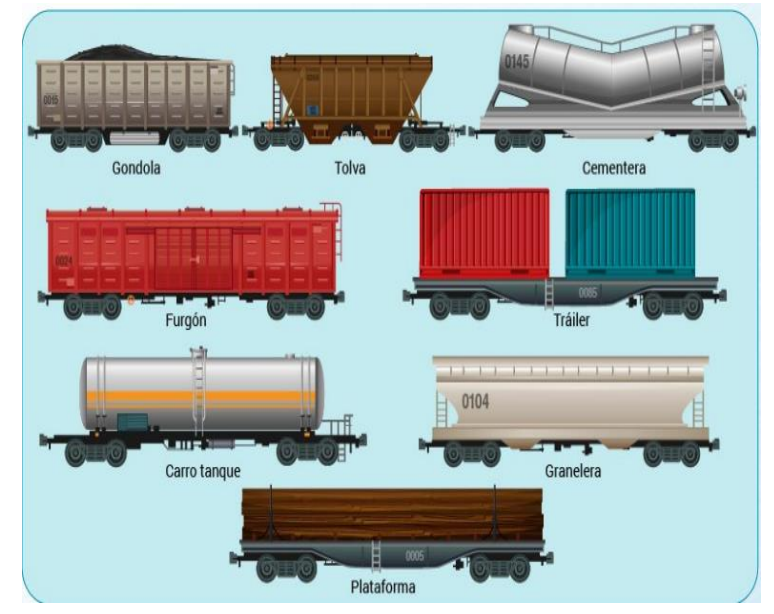

Fig. 3. Railroad transportation [6].

The speed of a freight train is about 70 kilometers per hour, is fixed by the general direction of railways for which takes two conditions [6].

The first: is established for each class of locomotive and is based by building system.

The second: is indicated by the state of the roads and the conditions of protection thereof.

The rate may be increased only with special authorization from the general direction of railroads.

Data on some containers to store items, restrictions of use and maximum total load

Table 1. Specifies the maximum load and dimensions of some transports containers currently on the market [6].

\begin{tabular}{|c|c|c|c|}
\hline Name & Length & Width & Height \\
\hline 12' steel dry box & $3.431 \mathrm{~m}$. & $2.352 \mathrm{~m}$. & $2.395 \mathrm{~m}$. \\
\hline 20' steel dry box & $5.900 \mathrm{~m}$. & $2.352 \mathrm{~m}$. & $2.395 \mathrm{~m}$. \\
\hline 40' steel dry box & $12.022 \mathrm{~m}$. & $2.352 \mathrm{~m}$. & $2.395 \mathrm{~m}$. \\
\hline Name & \multicolumn{2}{|c|}{ Maximum load } \\
\hline 20' dry & \multicolumn{2}{|c|}{$47,782 \mathrm{~kg}}$. \\
\hline 40' dry & \multicolumn{2}{c|}{$57,550 \mathrm{~kg}}$. \\
\hline 40' high cube
\end{tabular}




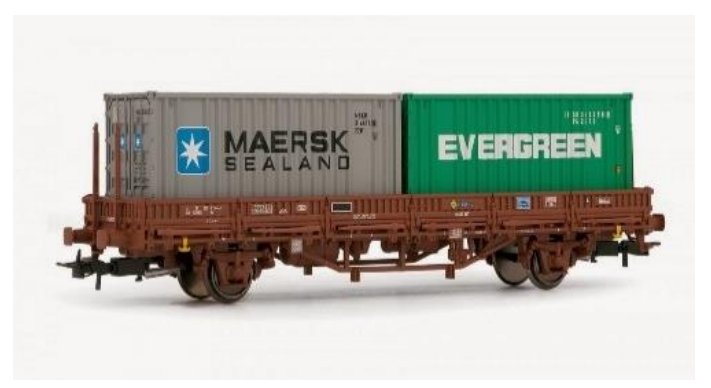

Fig. 4. Container [6].

\section{Kind of Wagons}

Boxcars: used to protect the goods from bad weather, theft and vandalism, the most used is the sliding walls.

Wagons edges: they are open boxes above and are used for transporting wood, scrap metal, etc.

Platforms: are used to transport vehicles, are considered high speed reaching $220 \mathrm{~km} / \mathrm{h}$, its structure is aluminum and not carbon steel.

Cage: used for transporting livestock.

Containerships: flatcars are having fasteners for containers.

Hoppers: are used to transport solid bulk cargo as aggregates, coal, minerals, etc. [1]

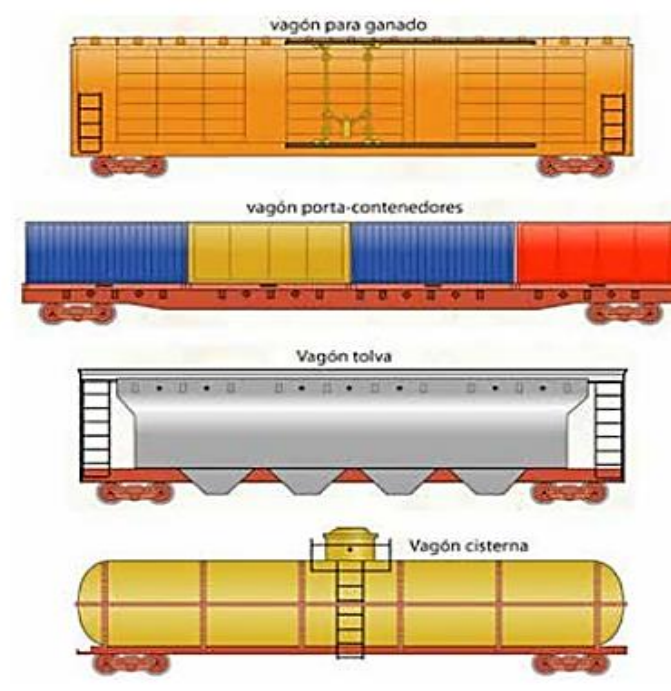

Fig. 5. Type of wagons. 


\section{$3 \quad$ Mode and Transport}

Transport modes are systems for mobilization, used in the transfer of goods from one source point to a destination.

The means of transport are the different physical elements used in the modes of transfer of goods to distribute in diverse location with far distance.

All modes and means of transport of goods, have advantages and disadvantages which must counter the logistics manager, to select the appropriate transport to the type and amount of cargo to be sent.

Table 2. Mode and meaning of transport train.

\begin{tabular}{|c|c|}
\hline Modes & \multicolumn{1}{c|}{ media } \\
\hline \multirow{2}{*}{ Railroad } & $\begin{array}{l}\text { railway train } \\
\text { They are special equipment with sealed wagons, chairs, and } \\
\text { container type wagons. Usually charge bulk goods such as coal, } \\
\text { grain, and platforms are also used for loading containers. }\end{array}$ \\
\hline
\end{tabular}

1. Diseños para comparar dos o más tratamientos

2. Diseños para estudiar el efecto de varios factores sobre una o más variables de respuesta 3. Diseños para la optimización
de procesos

4. Diseños robustos

5. Diseños de mezclas
Diseño completamente al azar Diseño de bloques completos al azar Diseño de cuadros latino y grecolatino

Diseños factoriales $2^{k}$

Diseños factoriales $3^{k}$

Diseños factoriales fraccionados $2^{k-p}$

$$
\begin{aligned}
& \text { Diseños para el modelo } \\
& \text { de primer orden }
\end{aligned}
$$

$$
\begin{aligned}
& \left\{\begin{array}{l}
\text { Diseños factoriales } 2^{k} \text { y } 2^{k-p} \\
\text { Diseño de Plakett-Burman } \\
\text { Diseño simplex }
\end{array}\right. \\
& \left\{\begin{array}{l}
\text { Diseño de composición central } \\
\text { Diseño de Box-Behnken } \\
\text { Diseños factoriales } 3^{k} \text { y } 3^{k-p}
\end{array}\right.
\end{aligned}
$$

$\{$ Arreglos ortogonales (diseños factoriales)

Diseño con arreglos interno y externo

Diseño simplex-reticular
Diseño simplex con centroide
Diseño con restricciones
Diseño axial

Fig. 6. Classification of experimental designs [8]. 
An algorithm for the optimization problem is represented by a primary quantity of items to save ( multiplied by the size of occupying, being of the same type), this result is subtracted from the total size of the container, if the space subtracts the container and if there are more items on hold, you choose to fill the container without triple the weight of all items, the weight of the container.

According [3] design of experiments is to plan and perform a set of tests in order to generate data that, when analyzed statistically, provide objective evidence to answer the questions raised by the experimenter situation.

\section{$4 \quad$ Process for Obtaining Citrus Products}

Harvesting methods are the same as those used for fruit eaten fresh, fruit is cut from the tree using ladders and is manually selected, the fruit is placed in baskets that can hold from 80 to 100 kilograms, to be carried to the transport vehicle [5].

Table 3. Major producers of citrus municipalities in Veracruz per hectare.

\begin{tabular}{|c|c|c|c|c|c|c|}
\hline County & $\begin{array}{c}\text { Naranja } \\
\text { (Ha.) }\end{array}$ & $\begin{array}{c}\text { Lima } \\
\text { persa } \\
\text { (Ha.) }\end{array}$ & $\begin{array}{c}\text { Mandarina } \\
\text { (Ha.) }\end{array}$ & $\begin{array}{c}\text { Tangerina } \\
\text { (Ha.) }\end{array}$ & $\begin{array}{c}\text { Toronja } \\
\text { (Ha.) }\end{array}$ & $\begin{array}{l}\text { Total } \\
\text { (Ha.) }\end{array}$ \\
\hline TEMAPACHE, VER. & 42,626 & 53 & 2,994 & 2,812 & 571 & 48,955 \\
\hline $\begin{array}{l}\text { MARTINEZ DE LA } \\
\text { TORRE, VER }\end{array}$ & 14,423 & 16,049 & 6 & 644 & 2,790 & 33,912 \\
\hline TIHUATLAN, VER. & 13,783 & 270 & 1,118 & 813 & 82 & 16,066 \\
\hline ATZALAN, VER. & 4,840 & 4,383 & 55 & 2,264 & 210 & 11,752 \\
\hline TUXPAN, VER. & 4,791 & 69 & 2,389 & 2,399 & 150 & 9,798 \\
\hline BENITO JUAREZ, VER. & 8,000 & 0 & 0 & 0 & 0 & 8,000 \\
\hline $\begin{array}{c}\text { CASTILLO DE TEAYO, } \\
\text { VER }\end{array}$ & 8,675 & 13 & 106 & 178 & 57 & 9,029 \\
\hline PAPANTLA, VER. & 9,870 & 664 & 160 & 50 & 203 & 10,947 \\
\hline $\begin{array}{l}\text { GUTIERREZ ZAMORA, } \\
\text { VER }\end{array}$ & 8,050 & 70 & 200 & 100 & 335 & 8,755 \\
\hline TECOLUTLA, VER. & 6,376 & 441 & 41 & 151 & 265 & 7,275 \\
\hline MISANTLA, VER. & 3,610 & 1,012 & 120 & 1,400 & 175 & 6,317 \\
\hline CHICONTEPEC, VER. & 3,646 & 0 & 0 & 0 & 0 & 3,646 \\
\hline $\begin{array}{c}\text { CAZONES DE } \\
\text { HERRERA, VER. }\end{array}$ & 3,191 & 16 & 58 & 189 & 250 & 3,704 \\
\hline TAMIAHUA, VER. & 1,911 & 20 & 439 & 332 & 4 & 2,705 \\
\hline TLAPACOYAN, VER. & 2,257 & 0 & 0 & 0 & 0 & 2,257 \\
\hline
\end{tabular}


Table 4. Main municipalities of citrus growers in Veracruz per tonne.

\begin{tabular}{|c|c|c|c|c|c|c|}
\hline County & $\begin{array}{l}\text { Naranja } \\
\text { (Ton.) }\end{array}$ & $\begin{array}{l}\text { Lima } \\
\text { persa } \\
\text { (Ton.) }\end{array}$ & $\begin{array}{l}\text { Mandarina } \\
\text { (Ton.) }\end{array}$ & $\begin{array}{l}\text { Tangerina } \\
\text { (Ton.) }\end{array}$ & $\begin{array}{l}\text { Toronja } \\
\text { (Ton.) }\end{array}$ & $\begin{array}{l}\text { Total } \\
\text { (Ton.) }\end{array}$ \\
\hline TEMAPACHE, VER. & 701,679 & 105 & 59,875 & 39,339 & 12,346 & 813,344 \\
\hline $\begin{array}{l}\text { MARTINEZ DE LA } \\
\text { TORRE, VER }\end{array}$ & 185,358 & 173,789 & 60 & 8,356 & 76,093 & 443,656 \\
\hline TIHUATLAN, VER. & 192,962 & 3,100 & 22,360 & 16,260 & 0 & 234,682 \\
\hline ATZALAN, VER. & 59,865 & 46,540 & 750 & 26,598 & 4,902 & 138,655 \\
\hline TUXPAN, VER. & 57,492 & 1,021 & 38,224 & 28,788 & 3,000 & 128,525 \\
\hline $\begin{array}{l}\text { BENITO JUAREZ, } \\
\text { VER. }\end{array}$ & 128,000 & 0 & 0 & 0 & 0 & 128,000 \\
\hline $\begin{array}{l}\text { CASTILLO DE } \\
\text { TEAYO, VER }\end{array}$ & 121,450 & 91 & 1,900 & 3,560 & 0 & 127,001 \\
\hline PAPANTLA, VER. & 98,400 & 5,500 & 0 & 0 & 4,000 & 107,900 \\
\hline $\begin{array}{c}\text { GUTIERREZ } \\
\text { ZAMORA, VER }\end{array}$ & 75,800 & 750 & 2,500 & 1,200 & 3,800 & 84,050 \\
\hline TECOLUTLA, VER. & 71,520 & 4,350 & 550 & 1,800 & 4,750 & 82,970 \\
\hline MISANTLA, VER. & 34,397 & 11,955 & 864 & 19,880 & 3,430 & 70,526 \\
\hline CHICONTEPEC, VER. & 58,336 & 0 & 0 & 0 & 0 & 58,336 \\
\hline $\begin{array}{c}\text { CAZONES DE } \\
\text { HERRERA, VER. }\end{array}$ & 51,056 & 150 & 1,200 & 3,780 & 0 & 56,186 \\
\hline TAMIAHUA, VER. & 26,754 & 296 & 7,016 & 3,981 & 80 & 38,127 \\
\hline TLAPACOYAN, VER. & 33,339 & 0 & 0 & 0 & 0 & 33,339 \\
\hline
\end{tabular}

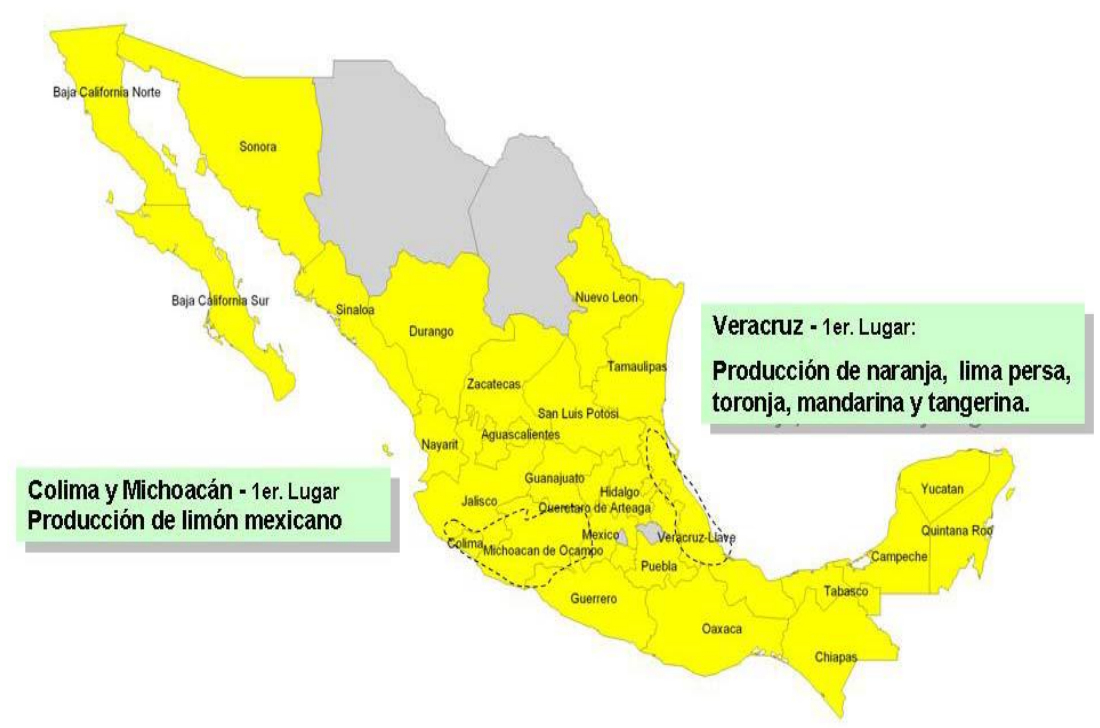

Fig. 7. Citrus production in Mexico. 
Veracruz leading citrus production in Mexico with a volume of 2.9 million tons. Followed by Tamaulipas with 638 thousand tons, and Mexican lime -producing states, Colima and Michoacan with 590,000 and 462,000 tons respectively [5].

Table 5. States citrus production in Mexico.

\begin{tabular}{|c|c|c|c|c|c|}
\hline No & ESTADO & $\begin{array}{l}\text { PRODUCCIÓN } \\
\text { (ton.) }\end{array}$ & No & ESTADO & $\begin{array}{l}\text { PRODUCCIÓN } \\
\text { (ton.) }\end{array}$ \\
\hline 1 & Veracruz & 2,987,973.65 & 15 & Quintana Roo & $42,044.45$ \\
\hline 2 & Tamaulipas & $638,262.49$ & 16 & Jalisco & $36,000.58$ \\
\hline 3 & Colima & $590,372.49$ & 17 & Baja California Sur & $32,624.72$ \\
\hline 4 & Michoacán & $462,999.49$ & 18 & Chiapas & $20,438.12$ \\
\hline 5 & $\begin{array}{l}\text { San Luís } \\
\text { Potosí }\end{array}$ & $436,915.80$ & 19 & Nayarit & $16,022.84$ \\
\hline 6 & Nuevo león & $399,739.22$ & 20 & Sinaloa & $14,344.80$ \\
\hline 7 & Oaxaca & $253,270.70$ & 21 & Baja California & $11,318.94$ \\
\hline 8 & Yucatán & $251,073.34$ & 22 & Morelos & $6,765.90$ \\
\hline 9 & Puebla & $225,595.90$ & 23 & Durango & $2,420.70$ \\
\hline 10 & Sonora & $191,208.30$ & 24 & México & $1,804.90$ \\
\hline 11 & Tabasco & $141,300.00$ & 25 & Querétaro & $1,415.50$ \\
\hline 12 & Guerrero & $85,816.56$ & 26 & Zacatecas & 260.00 \\
\hline 13 & Hidalgo & $58,731.05$ & 27 & Aguascalientes & 124.00 \\
\hline 14 & Campeche & $58,267.56$ & 28 & Guanajuato & 44.60 \\
\hline
\end{tabular}

\section{$5 \quad$ Problem Packaging of Objects in Containers: Bin Packing}

The problem of distribution of objects in containers, in English Bin Packing is a classic problem of NP- hard combinatorial optimization . Because Bin Packing is a highly complex problem can not be solved big cases using an exact algorithm. An optimal solution can be found by considering all ways to make a partition of $n$ objects in a subset of size $n$ or smaller.

\section{Algorithms for Optimization Problems and Generic Packaging Containers}

$\mathrm{O}(\mathrm{g}(\mathrm{x}))=\left\{\begin{array}{l}\mathrm{F}(\mathrm{x}): \text { Exists } \mathrm{c}, \mathrm{x}_{0}>0 \text { such as } \\ \forall \mathrm{x} \geq \mathrm{x}_{0}: 0 \leq|\mathrm{f}(\mathrm{x})| \leq \mathrm{c}|\mathrm{g}(\mathrm{x})|\end{array}\right\}$

A container $\mathrm{f}(\mathrm{x})$ belongs to items $\mathrm{g}(\mathrm{x})$, when there is a positive constant $\mathrm{c}$ such that from a quantity of the items $\mathrm{x} 0, \mathrm{f}(\mathrm{x})$ not on passing containers $(\mathrm{x})$, means that the function $\mathrm{f}$ is less aga from a given value by a constant factor. 
Generic algorithm (GA) .

Assuming a search space $\mathrm{x}$, and the function:

$\mathrm{f}: \mathrm{x} \rightarrow \mathrm{R}$

The general problem is to $\min \mathrm{f}$

$\mathrm{X} \varepsilon \mathrm{X}$

Where $\mathrm{x}$ is the vector of decision variables and $\mathrm{f}$ is the objective function. This methodology consists of three major phases . The first is the generation population ( chromosomes ); the second is the recombination of these chromosomes or crossover criterion and the last mutation. Finding the best solution it is guided by the results and evaluation of the objective function $\mathrm{f}$ for each of the individuals generated . Finally, based on this evaluation, the chromosome with higher value of $\mathrm{f}$ represents the best solution [4].

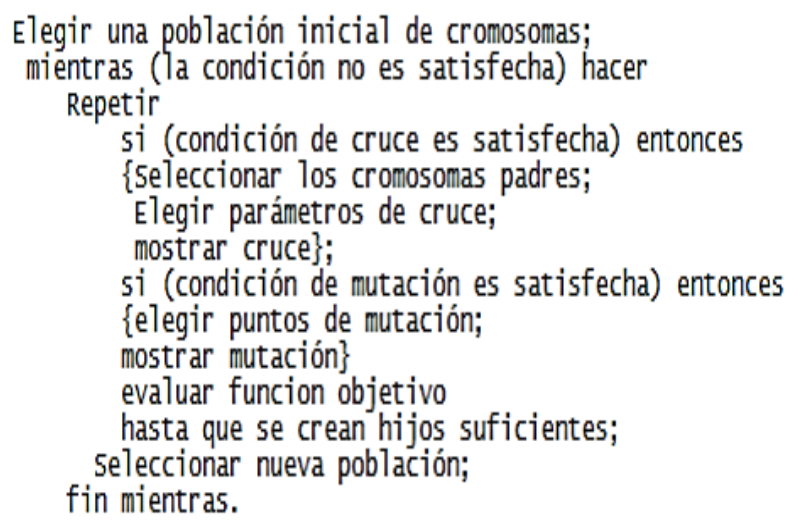

Fig. 8. Description of the operation of AG [4].

Program in Matlab of the generic algorithm for the problem of packaging in containers. By using a container crossing.

function [pob bins]=cruce(numind,nuevabins,nuevapob,pesos,capacidad)

Cruce de contenedores

bjacm=[];

for i3=1:size(listap,2)

[listac indc] $=\operatorname{sort}(($ hijo(1:numbinsaux,numobj+2))',2,'ascend');

for $\mathrm{i} 4=1$ :size $($ listac, 2$)$

if (listac(i4)+listap(i3)) < capacidad

hijo(indc(i4),hijo(indc(i4),numobj+1)+1)=vecobj(indp(i3));

hijo(inde(i4),numobj+1)=hijo(inde(i4),numobj+1)+1;

hijo(indc(i4),numobj+2)=hijo(inde(i4),numobj+2)+listap(i3);

objacm=[objacm vecobj(indp(i3))];

listac(i5)=listac(i5)+listap(i3); 
break

end

end

end

vecobj=setdiff(vecobj,objacm);

if $\operatorname{size}(\operatorname{vecobj}, 2)^{\sim}=0$

indbin $=1$;

numbinsaux $=$ numbinsaux +1 ;

for $\mathrm{i} 3=1$ :size $($ vecobj, 2$)$

if (hijo(numbinsaux,numobj+2)+pesos(vecobj(i3)))>capacidad

numbinsaux $=$ numbinsaux +1 ;

indbin $=1$;

end

hijo(numbinsaux,indbin)=vecobj(i3);

hijo(numbinsaux,numobj+1)=indbin;

hijo(numbinsaux,numobj+2)=hijo(numbinsaux,numobj+2)+pesos(vecobj(i3));

indbin=indbin +1 ;

end

end

nuevapob(:,:,conthijo)=hijo;

nuevabins (conthijo)=numbinsaux;

conthijo=conthijo+1;

end

end

pob=nuevapob;

bins=nuevabins;

In this code is explain each iteration related with the correct location of objects in a transport model by train.

Table 6. Iteration 1.Testing generic algorithm: Crossing container.

\begin{tabular}{|c|c|c|c|c|}
\hline $\begin{array}{c}\text { Number of } \\
\text { executions }\end{array}$ & Data & Result & \% Total & $\begin{array}{c}\text { Probability } \\
\text { Overload }\end{array}$ \\
\hline 1 & $0,1,1,1,1$ & 39.8 & 37.1 & 0.374 \\
\hline 2 & $1,0,0,0,1$ & 24.4 & 22.8 & 0.226 \\
\hline 3 & $1,1,0,0,0$ & 22.4 & 20.9 & 0.208 \\
\hline 4 & $0,1,0,0,0$ & 20.4 & 19.0 & 0.189 \\
\hline Sum & & 107 & 99.8 & 0.998 \\
\hline
\end{tabular}


Table 7. Executions in Matlab program.

\begin{tabular}{|c|c|c|c|}
\hline $\begin{array}{l}>> \\
\text { bins] }=\text { cruce }(0,1,1,1,1 \\
) \\
\text { pob }= \\
1 \\
\text { bins }= \\
1\end{array}$ & $\begin{array}{l}>> \\
\text { bins] }=\text { cruce }(1,0,0,0,1 \\
\text { pob }= \\
0 \\
\text { bins }= \\
0\end{array}$ & $\begin{array}{l}>> \\
\text { bins] }=\text { cruce }(0,1,0,0,0 \\
) \\
\text { pob }= \\
0 \\
\text { bins }= \\
1\end{array}$ & $\begin{array}{l}>> \\
\text { bins }]=\text { cruce }(1,1,0,0,0 \\
\text { pob }= \\
\quad 0 \\
\text { bins }= \\
\quad 1\end{array}$ \\
\hline
\end{tabular}

Table 8. Iteration 2.

\begin{tabular}{|c|c|c|c|c|}
\hline $\begin{array}{c}\text { Number of } \\
\text { executions }\end{array}$ & Data & Result & \% Total & $\begin{array}{c}\text { Load } \\
\text { problems }\end{array}$ \\
\hline 1 & $1,1,0,0,0$ & 22.1 & 31.9 & 0.319 \\
\hline 2 & $0,1,0,0,0$ & 20.4 & 28.9 & 0.289 \\
\hline 3 & $1,0,0,1,1$ & 18.4 & 26.1 & 0.261 \\
\hline 4 & $0,1,1,0,1$ & 9.8 & 13.9 & 0.139 \\
\hline Suma & & 70.4 & 100.8 & 1.008 \\
\hline
\end{tabular}

Table 9. Executions in Matlab program.

\begin{tabular}{|c|c|c|c|}
\hline $\begin{array}{l}>> \\
\text { bins }]=\text { cruce }(1,1,0,0,0 \\
) \\
\text { pob }= \\
0 \\
\text { bins }= \\
1\end{array}$ & $\begin{array}{l}\text { [pob } \\
\text { bins] }=\operatorname{cruce}(0,1,0,0,0 \\
\text { pob }= \\
\quad 0 \\
\text { bins }= \\
\quad 1\end{array}$ & $\begin{array}{l}\text { [pob } \\
\text { bins] }=\text { cruce }(1,0,0,1,1 \\
\text { pob }= \\
0 \\
\text { bins }= \\
\quad 0\end{array}$ & $\begin{array}{l}\text { [pob } \\
\text { bins]=cruce }(0,1,1,0,1 \\
\text { J } \\
\text { pob }= \\
1 \\
\text { bins }= \\
\quad 1\end{array}$ \\
\hline
\end{tabular}

Table 10. Iteration 3.

\begin{tabular}{|c|c|c|c|c|}
\hline $\begin{array}{c}\text { Number of } \\
\text { executions }\end{array}$ & Data & Result & \% Total & $\begin{array}{c}\text { Load } \\
\text { problems }\end{array}$ \\
\hline 1 & $1,0,0,0,1$ & 24.4 & 22.8 & 0.228 \\
\hline 2 & $0,1,1,1,1$ & 39.8 & 37.1 & 0.371 \\
\hline 3 & $1,1,0,0,0$ & 22.4 & 20.9 & 0.209 \\
\hline 4 & $0,1,0,0,0$ & 20.4 & 19.0 & 0.190 \\
\hline Suma & & 107 & 99.8 & 0.998 \\
\hline
\end{tabular}


Table 11. Executions in Matlab program.

\begin{tabular}{|c|c|c|c|}
\hline $\begin{array}{l}>> \\
\text { bins] }=\text { cruce }(1,0,0,0,1 \\
\text { pob }= \\
0 \\
\text { bins }= \\
0\end{array}$ & $\begin{array}{l}\text { [pob } \\
\text { bins]=cruce }(0,1,1,1,1 \\
\text { J } \\
\text { pob }= \\
1 \\
\text { bins }= \\
1\end{array}$ & $\begin{array}{l}\text { [pob } \\
\text { bins] }=\text { cruce }(1,1,0,0,0 \\
\text { J } \\
\text { pob }= \\
0 \\
\text { bins }= \\
1\end{array}$ & $\begin{array}{l}\text { [pob } \\
\text { bins] }=\text { cruce }(0,1,0,0,0 \\
\text { J } \\
\text { pob }= \\
0 \\
\text { bins }= \\
1\end{array}$ \\
\hline
\end{tabular}

Table 12. Iteration 4.

\begin{tabular}{|c|c|c|c|c|}
\hline $\begin{array}{c}\text { Number of } \\
\text { executions }\end{array}$ & Data & Result & \% Total & $\begin{array}{c}\text { Load } \\
\text { problems }\end{array}$ \\
\hline 1 & $1,1,0,0,0$ & 22.4 & 31.5 & 0.315 \\
\hline 2 & $0,1,0,0,0$ & 20.4 & 28.7 & 0.287 \\
\hline 3 & $1,0,0,1,1$ & 18.4 & 25.9 & 0.259 \\
\hline 4 & $0,1,1,0,1$ & 9.8 & 13.8 & 0.138 \\
\hline Suma & & 71 & 99.9 & 0.999 \\
\hline
\end{tabular}

Table 13. Executions in Matlab program.

\begin{tabular}{|c|c|c|c|}
\hline $\begin{array}{l}\text { [pob } \\
\text { bins] }=\text { cruce }(1,1,0,0,0 \\
\text { p pob }= \\
0 \\
\text { bins }= \\
\quad 1\end{array}$ & $\begin{array}{l}\text { [pob } \\
\text { bins] }=\text { cruce }(0,1,0,0,0 \\
\text { J } \\
\text { pob }= \\
\quad 0 \\
\text { bins }= \\
\quad 1\end{array}$ & $\begin{array}{l}\text { [pob } \\
\text { bins] }=\text { cruce }(1,0,0,1,1 \\
\text { ) } \\
\text { pob }= \\
0 \\
\text { bins }= \\
0 \\
0\end{array}$ & $\begin{array}{l}\text { [pob } \\
\text { bins] }=\text { cruce }(0,1,1,0,1 \\
\text { J } \\
\text { pob }= \\
1 \\
\text { bins }= \\
\quad 1\end{array}$ \\
\hline
\end{tabular}

Table 14. Iteration 5.

\begin{tabular}{|c|c|c|c|c|}
\hline $\begin{array}{c}\text { Number of } \\
\text { executions }\end{array}$ & Data & Result & \% Total & $\begin{array}{c}\text { Load } \\
\text { problems }\end{array}$ \\
\hline 1 & $1,0,0,0,1$ & 24.4 & 22.8 & 0.374 \\
\hline 2 & $0,1,1,1,1$ & 39.8 & 37.1 & 0.226 \\
\hline 3 & $1,1,0,0,0$ & 22.4 & 20.9 & 0.208 \\
\hline 4 & $0,1,0,0,0$ & 20.4 & 19.0 & 0.189 \\
\hline Suma & & 107 & 99.8 & 0.998 \\
\hline
\end{tabular}


Table 15. Executions in Matlab program.

\begin{tabular}{|c|c|c|c|}
\hline $\begin{array}{l}>> \\
\text { bins] }=\operatorname{cruce}(1,0,0,0,1) \\
\text { pob }= \\
0 \\
\text { bins }= \\
0\end{array}$ & $\begin{array}{l}>> \\
\text { bins]=cruce }(0,1,1,1,1) \\
\text { pob }= \\
\quad 1 \\
\text { bins }= \\
\quad 1\end{array}$ & $\begin{array}{l}>> \\
\text { bins]=cruce }(1,1,0,0,0) \\
\text { pob }= \\
0 \\
\text { bins }= \\
\quad 1\end{array}$ & $\begin{array}{l}>>\quad \text { [pob } \\
\text { bins]=cruce }(0,1,0, \\
0,0) \\
\text { pob }= \\
0 \\
\text { bins }= \\
1\end{array}$ \\
\hline
\end{tabular}

Table 16. Capacity and containers are listed in the following table.

\begin{tabular}{|c|c|c|c|c|c|}
\hline & Container 1 & Container 2 & Container 3 & Container 4 & Container 5 \\
\hline $\begin{array}{c}\text { Capacity } \\
\text { (tons) }\end{array}$ & 2 & 2.4 & 3 & 4 & 4.4 \\
\hline
\end{tabular}

The capacity of all containers is determined as follows :
$(1,1,1,1,1)=41.8 \quad(1,1,1,1,0)=29.4 \quad(1,1,1,0,1)=29.8$
$(1,1,1,0,0)=7.4 \quad(1,1,0,1,1)=38.8 \quad(1,1,0,1,0)=8.4$
$(1,1,0,0,1)=8.8 \quad(1,1,0,0,0)=22.4 \quad(1,0,1,1,1)=39.4$
$(1,0,1,1,0)=9 \quad(1,0,1,0,1)=9.4 \quad(1,0,1,0,0)=23$
$(1,0,0,1,1)=18.4 \quad(1,0,0,1,0)=24 \quad(1,0,0,0,1)=24.4$
$(1,0,0,0,0)=20 \quad(0,0,0,0,0)=18 \quad(0,1,1,1,1)=39.8$
$(0,1,1,1,0)=9.4 \quad(0,1,1,0,1)=9.8 \quad(0,1,1,0,0)=23.4$
$(0,1,0,1,1)=18.8 \quad(0,1,0,1,0)=24.4 \quad(0,1,0,0,1)=24.8$
$(0,1,0,0,0)=20.4 \quad(0,0,1,1,1)=19.4 \quad(0,0,1,1,0)=25$
$(0,0,1,0,1)=25.4 \quad(0,0,1,0,0)=21 \quad(0,0,0,1,1)=34.4$
$(0,0,0,1,0)=22 \quad(0,0,0,0,1)=22.4$

Results are calculated as follows:

The values 18 and 8 only considered the containers 4 and 5 have a storage capacity of relatively equal [8].

\section{Examples:}
$(1,1,1,1,1)=2+2.2+3+4+4.4+18+8=41.8$,
$(0,1,0,1,0)=2,4+4+18=24.8$,
$(0,0,1,1,0)=3+4+18=25$.

\section{Conclusions and Future Work}

The results obtained on the problems of packaging containers experimentally demonstrate that combinatorial problems that are untreatable or difficult to solve with an exhaustive 
search or classical techniques, can be solved satisfactorily with evolutionary algorithms that are modified appropriately.

An advantage of these processes is that it is not necessary to know the description of the problem, a way to solve the problem is with the implementation of the generic algorithm, but is complicated adapting techniques to find an optimal solution, and thats where the generic algorithms have found an important area of solution.

In future work we should be studied more chromosomes of the cell or humans to solve problems with the help of artificial intelligence.

\section{References}

1. Berbey, A., et al.: Trenes: Material rodante del transporte ferroviario.

2. Caliz, E.: Validación de la calidad de las soluciones obtenidas en la tesis de maestría optimización integral del problema de carga de contenedores tridimensionales (2012)

3. Gutiérrez, A., et al.: Análisis y diseño de experimentos. Tercera edición, Editorial Mc Graw Hill (2013)

4. Hernández, N., et al.: Introducción a Matlab para Resolver Problemas de Ingeniería Aplicando Algoritmos Genéticos (2013)

5. Licona, J.: Estudio de Mercado para identificación de necesidades de infraestructura logística para la comercialización de jugo de cítricos en Veracruz (2009)

6. Mata, G., et al.: Un problema de tipo Bin Packing (2011)

7. Reglamento General de Ferrocarriles. Aprobado por Decreto $N^{\circ} 90.325$ del 12 de Setiembre de 1936 y actualizado al 31 de Diciembre de 1995

8. Vidal, A.: Algoritmos heurísticos de optimización. Universidad de Santiago de Compostela, Facultad de Matemáticas Master en Técnicas Estadísticas (2013) 